\title{
Pierre Sciobéret en 1857-1864
}

Du Moléson au Caucase, en passant par Saint-Gall et la NZZ

Jean-Christophe Emmenegger

\section{CpenEdition}

Journals

Édition électronique

URL : http://journals.openedition.org/edl/443

DOI : $10.4000 /$ edl. 443

ISSN : 2296-5084

Éditeur

Université de Lausanne

Édition imprimée

Date de publication : 15 septembre 2009

Pagination : 275-294

ISBN : 978-2-940331-20-8

ISSN : 0014-2026

Référence électronique

Jean-Christophe Emmenegger, «Pierre Sciobéret en 1857-1864 », Études de lettres [En ligne], 2-3 |

2009, mis en ligne le 15 septembre 2012, consulté le 19 décembre 2020. URL : http://

journals.openedition.org/edl/443; DOI : https://doi.org/10.4000/edl.443 


\section{PIERRE SCIOBÉRET EN 1857-1864 DU MOLÉSON AU CAUCASE, EN PASSANT PAR SAINT-GALL ET LA $N Z Z$}

En comparaison du grand nombre d'écrivains occidentaux ayant voyagé en Orient, suivant le tour méditerranéen classique, un petit nombre a poussé jusque dans l'Orient particulier du Caucase, au XIX ${ }^{\mathrm{e}}$ siècle. Selon ce même rapport, les écrivains d'expression française qui ont laissé des traces de leur périple ou de leur séjour au Caucase ne sont pas non plus légion. Parmi eux, l'on trouve Pierre Sciobéret, qui paraît avoir été le seul écrivain romand et peut-être le seul Suisse à rapporter de son séjour au Caucase une ouvre de fiction, Abdallah Schlatter ou les aventures d'un Suisse au Caucase. Mais ce court roman d'aventures a curieusement disparu des histoires littéraires, qui nous présentent encore Pierre Sciobéret comme un parangon de la littérature romande de type régionaliste. Il pourrait compter, au contraire, comme un des regards les plus originaux dans le contexte colonialiste de l'époque.

Quand bien même Mikhaïl Miansarov a recensé, dans sa bibliographie commentée du Caucase jusqu'en 1874, quatre cent cinquante relations de voyage (en comptant dans ce nombre les traductions) écrites en différentes langues, dont cent quarante-trois ont été publiées entre les années $1800-1840^{1}$, et des milliers de textes de tout genre, il n'est pas question ici d'aborder les différentes formes d'écriture liées à cette recension; on garde en réserve l'explication des situations et des modalités du discours,

I. Bibliographia Caucasica et Transcaucasica. Essai d'une bibliographie systématique relative au Caucase, à la Transcaucasie et aux populations de ces contrées, par M. Miansarof, t. I, sections I et II, St.-Pétersbourg, J. Bakst, Hohenfelden et Comp., 1874-1876, p. 320-388. Cité par A. Schnetzer, «Schweizer im Kaukasus: Forscher, Alpinisten und andere Reisende (1801-1939)», p. 383. 
et on ne cherche pas davantage à comprendre la dynamique présente en remuant le passé. On prend le cas d'un laissé-pour-compte: le Suisse Pierre Sciobéret (1830-1876), qui avait «décrit les rives de la mer Noire, et les montagnes du Caucase " ${ }^{2}$, et publié à Lausanne un récit intitulé Abdallah Schlatter ou les curieuses aventures d'un Suisse au Caucase ${ }^{3}$. Dans la plus grande indifférence générale, semble-t-il, jusqu'à nos jours, la période de la vie de Sciobéret qui se rapporte au Caucase et surtout la trace qui en résulte sous la forme du récit intitulé Abdallah Schlatter pâtissent de l'ordre établi en République des Lettres, contrairement aux œuvres "rustiques» du même auteur.

Car Sciobéret est surtout connu - ou l'a été - pour ses Scènes de la vie gruyérienne (1854), Scènes de la vie du peuple montagnard fribourgeois (1856) et autres nouvelles "rustiques", d'abord publiées en revues, puis recueillies posthumément sous les appellations évocatrices de Scènes de la Vie champêtre (1882) et Nouvelles Scènes de la Vie champêtre (1884) ${ }^{4}$. A ce titre, il n'a pas cessé, jusque dans la récente Histoire de la littérature en Suisse romande ${ }^{5}$ d'être considéré comme un écrivain "populaire", tout au plus de second rang ou même médiocre, selon Gonzague de Reynold ${ }^{6}$. Ce jugement repose exclusivement sur la lecture des textes "rustiques» ou plutôt sur les effets du code limité qui a prévalu à leur interprétation jusqu'à nos jours ${ }^{7}$. Un indice de l'insuffisance de cette

2. F. Rime, «Pierre Sciobéret», p. 109.

3. P. Sciobéret, Abdallah Schlatter ou les curieuses aventures d'un Suisse au Caucase.

4. "Le vrai Sciobéret se trouve dans les Scènes de la vie champêtre", assène E. Dévaud ("Les écrivains gruyériens de L’Emulation», p. 725). Voir aussi L. Bornet, "Revue bibliographique: Scènes de la vie gruyérienne par M. P. Sciobéret»; M. Buchon, "Causeries littéraires»; G. de Reynold, "Les Poètes de la Gruyère»; P. Bondallaz, «Le Mouvement littéraire en pays fribourgeois vers 1850 », etc.

5. D. Maggetti, «Une littérature populaire?», p. 156.

6. Voir sa «Préface» à la thèse de R. Loup, Un Conteur Gruyérien, Pierre Sciobéret, 1830-1876, p. VIII.

7. Sauf exception, les études thématiques et les histoires littéraires qui suivent omettent donc systématiquement de mentionner Abdallah Schlatter pour pouvoir mieux lisser le modèle de l'écrivain régionaliste, en l'ouvrant parfois au monde mais en oubliant les nouvelles de jeunesse écrites en voyage et les écrits du polémiste violent, défendant une certaine idée de l'art contre l'étouffement provincial et bourgeois de tous les temps, dont l'expression a des tonalités rimbaldiennes avant l'heure: Ph. E. Godet, Histoire littéraire de la Suisse française, p. 536-537; V. Rossel et H.-E. Jenny, Histoire de la littérature suisse, t. 2, p. 224-225; R. Zellweger, Les Débuts du roman rustique: Suisse, Allemagne, France, 1836-1856, p. 184, 199, 210 et 211; J. Humbert, La Poésie au 
analyse provient de ce que les qualités ou les défauts de l'écrivain se trouvent symétriquement opposés chez les lecteurs: tantôt son style est "toujours correct, se distingue par l'élégance et la sobriété» ${ }^{8}$, tantôt au contraire «il n'a ni assez d'aisance ni assez de distinction; il n'est pas toujours correct " ${ }^{9}$. De tels jugements de valeur pourraient refléter le partage entre de distingués amateurs de la culture française, et des Fribourgeois forcés d'expérimenter l'influence de l'aire germanique, qui demeure à ce moment-là "un passage obligé pour la plupart des jeunes gens soucieux de poursuivre leurs études ${ }^{10}$. La condition et l'appartenance politique des commentateurs, libéraux-radicaux versus conservateurs-catholiques, région de Gruyères versus ville de Fribourg, classe bourgeoise versus classe ouvrière, ont pu également jouer un rôle ${ }^{11}$. Cela n'explique pas comment ils ont pu rester presque tous insensibles à l'exotisme de la dernière ouvre en prose de l'auteur:

Recueil des souvenirs de l'exil, fabuleux récit des Mille et une nuits, pages d'histoire et de géographie, livre d'adieu aux lettres et chant du cygne, Abdallah Schlatter ajoute à ces raisons d'intéresser l'agrément d'une lecture facile et captivante ${ }^{12}$.

L'intérêt n'a pas eu lieu et le clou fut enfoncé: «Sciobéret n’est pas un grand écrivain, mais il est un type représentatif de l'esprit gruyérien au milieu du siècle dernier " ${ }^{13}$... Sauf qu'il est le seul "esprit gruérien»

Pays de Gruyère, p. 61-65; P. Kohler, G. Guisan et E. Pidoux, Histoire de la littérature française, vol. 3, p. 769-770; M. Gremaud, «Pierre Sciobéret (1830-1876). Conteur et romancier gruyérien", etc. La mise à l'index de Abdallah Schlatter a porté ses fruits dès lors qu'il s'était agi de dresser une bibliographie "complète» des œuvres de l'écrivain, dans l'Etude historique sur la Littérature Fribourgeoise depuis le moyen âge à la fin $d u$ $X I X^{\mathrm{e}}$ siècle de l'abbé F. J. Brülhart, ou de présenter l'auteur à une large audience, dans l'article consacré à «P. Sciobéret» de l'Encyclopédie du Canton de Fribourg (t. 2, p. 501).

8. C. Ayer, «Notice biographique», p. XXVI.

9. V. Rossel, Histoire littéraire de la Suisse romande des origines à nos jours, p. 609.

Io. S. de Reyff, "L'“idéal favori” d'Alexandre Daguet ou les pages littéraires de L'Emulation", p. 31.

II. Pour les idéologies dans ce contexte: S. Rossier: "G. de Reynold et L'Emulation, une lecture orientée», p. 131-140; «Robert Loup et Jean Humbert: deux thèses reynoldiennes", p. 141-151.

I2. R. Loup, Un Conteur Gruyérien, Pierre Sciobéret, 1830-1876, p. 153.

I3. J. Niquille, "Robert Loup [etc.] ", p. 91. Il s'agit d'un compte-rendu de la thèse de R. Loup, Pierre Sciobéret, 1830-1876. Sa vie. Son æuvre. 
pour embrasser la région du Caucase au point d'en tirer une ouvre littéraire, l'un des rares Suisses romands à se consacrer au sujet ${ }^{14}$, l'un des rares écrivains d'expression française et vraisemblablement le seul Suisse à représenter la géographie du Caucase dans une œuvre de fiction au XIX ${ }^{\mathrm{e}}$ siècle...

Par ailleurs, cette belle définition d'Abdallah Schlatter peut induire en erreur. En particulier la première proposition, « recueil des souvenirs de l'exil», qui ferait croire à tort à un contenu purement autobiographique. L'idée que ce «récit romancé» posséderait "de nombreux traits autobiographiques" remonte loin ${ }^{15}$ et a été reprise récemment ${ }^{16}$. Mais des recherches plus poussées tendent à nuancer cette assertion sinon à démontrer le contraire. En effet, Abdallah Schlatter renvoie à un commis voyageur, originaire de Saint-Gall, ayant vécu à la même époque que Sciobéret, et qui répond au nom authentique de... Abdullah Schlatter. Ici, c'est bien de lire au pied de la lettre.

Abdallah Schlatter mérite donc l'attention pour deux raisons: d'abord, il se pourrait que cette ouvre éclairât sous un jour nouveau l'écriture d'un auteur déjà considéré comme «régionaliste» par ses contemporains; puis, en-dehors d'un intérêt local, elle recèle un potentiel d'interprétation destiné aux recherches interdisciplinaires et en particulier au domaine de la littérature comparée. Sciobéret ne côtoie-t-il pas, à Tiflis ${ }^{17}$, à peu de mois ou d'années près, Alexandre Dumas, Arthur de Gobineau et tant d'autres auteurs moins connus de viatiques, peut-être le même personnel consulaire, les mêmes expatriés, les mêmes indigènes, à tout le moins le même environnement qu'eux?

I4. Voir la recension effectuée par A. Schnetzer, «Schweizer im Kaukasus: Forscher, Alpinisten und andere Reisende (1801-1939)», p. 383-432.

15. Par exemple, E. Dévaud, «Les Ecrivains gruyériens de L’Emulation», p. 748.

I6. F. Rime, "Pierre Sciobéret», p. 108. M. Gremaud et J. Cesa, Pierre Sciobéret, Colin l'armailli. Le Regain. Sur la trace de Pierre Sciobéret (1830-1876), p. 6 et 158.

I7. Aujourd'hui Tbilissi, capitale de la Géorgie. 
Moléson - Tiflis

Pierre Sciobéret, écrivain et homme politique ${ }^{18}$, est né au pied du Moléson, dans le village de Gruyères La Tour-de-Trême, près de Bulle. Il bénéficie d'une éducation auprès des Jésuites du collège Saint-Michel à Fribourg (1843-1848), mais se range du côté des libéraux-radicaux contre le régime catholique-conservateur, lors des événements révolutionnaires de 1848, qui touchent de plein fouet la ville catholique de Fribourg, entourée de cantons protestants. C'est ce qui lui vaudra, après un séjour universitaire à Berlin (1849-1852) et un professorat à Fribourg (1852-1856), lorsque le parti conservateur revient au pouvoir, de choisir l'exil à Odessa puis en Transcaucasie, où sept années de préceptorat l'attendent (1857-1864).

Le 22 avril 1857, Sciobéret obtient son sésame pour la Russie, valable pour une durée indéterminée. La trace en est consignée dans le Registre des passeports fribourgeois ${ }^{19}$. La date de son départ reste imprécise (un soir de printemps) tout comme l'est la manière dont il a déniché ce préceptorat: souvenirs d'anciens émigrés, lettres de précepteurs ou de gouvernantes, demandes ou offres d'emploi publiées dans les Feuille[s] d'Avis cantonales, bureaux de placement avec leurs filiales à l'étranger sont les moyens les plus courants d'assurer le placement des volontaires à l'étranger ${ }^{20}$. Plusieurs Fribourgeois avaient précédé Sciobéret à Odessa: "Nous ne pouvons citer les noms des instituteurs et des institutrices en grand nombre, des cantons de Vaud, de Genève, de Neuchâtel, de Fribourg et de Berne, qui ont été ou sont encore placés dans des familles du midi de la Russie ${ }^{21}$.»

I8. J. Humbert, «Sciobéret, Pierre», p. 501. Les données biographiques sont tirés principalement des sources suivantes: C. Ayer, «Notice Biographique», p. V à XXX; E. Rambert, «Introduction», p. V à XV; J. Reichlen, «Pierre Sciobéret», planche VII et p. 11 et suiv.; J. Sterroz, "Les Poètes de la Gruyère: choix de leurs poésies ", p. 89 et suiv.; M. Gremaud et J. Cesa, Pierre Sciobéret, Colin l'armailli. Le regain. Sur la trace de Pierre Sciobéret (1830-1876), p. 127 et suiv.

19. AEF, Registre des passeports du canton de Fribourg (CH AEF DPc II 14), passeport pour Odessa/ Russie le 22 avril 1857.

20. Voir A. Maeder, Gouverneurs et précepteurs neuchâtelois dans l'empire russe (1800-1890), p. 65-71; A.-S. de Weck, Un Mouvement migratoire insoupçonné: les Fribourgeoises en Europe centrale et orientale (1860-1914).

2I. L. Vulliemin, «Les Suisses en Russie», p. 239. Le mouvement inverse est moins connu: y'a-t-il eu des contacts entre Fribourgeois et voyageurs russes, en excluant 
A Odessa, la ville fondée par Catherine II, en 1794, Sciobéret est d'abord engagé comme gouverneur, enseignant le français, le latin et l'allemand dans la famille d'un certain amiral Barkowski: "c'était l'affaire de trois à quatre heures de leçons par jour, tout au plus» ${ }^{22}$. D'abord, il se sent gai à cause de sa nouvelle situation:

Depuis que je suis en Russie, j'ai rajeuni, j’ai retrouvé ma gaîté, mon insouciance, je dirai même ma présomption d'autrefois; je respire plus à l'aise, je n'éprouve plus ce sentiment indéfinissable qui vous prend quand vous vous sentez glisser sur un crapaud ou sur une vipère ${ }^{23}$.

C'est un classique du voyageur et peut-être aussi du conquérant, ce sentiment de liberté éprouvé sur de nouveaux territoires, et Alexandre Dumas (père) le partage en mots curieusement semblables avec l'une de ses maîtresses:

Je suis rajeuni de dix ans comme force et je dirai presque comme visage. [...] La bonne chose que cette liberté de faire ce que l'on veut, de se mettre ce que l'on veut, d'aller où l'on veut! ${ }^{24}$

Mais Sciobéret n'a pas les mêmes facilités que Dumas, il est à Odessa pour travailler, très vite la nostalgie le rattrape, et il la repousse en imaginant pouvoir vivre un jour comme il l'entend, en poète:

Si tu tiens à connaître mes projets pour l'avenir, les voici. Dès que je le pourrai décemment et que l'occasion s'en présentera, je me lancerai vers le nord, au Caucase ou en Sibérie. C'est une expédition dangereuse, il est vrai, car on n'en revient pas toujours; on vous tue quelquefois au lieu de vous payer, mais, quand on réussit, on y réalise de

Tolstoï qui passe près de Fribourg sans s'y arrêter et Alexandre Herzen qui y réside quelque temps? En 1856, séjourne pourtant à Fribourg la jeune écrivaine russe Ljudmila Petrovna Šelgunova née Michaélis (1832-1901), après six semaines passées en cure à Ems (St. Moritz) : elle peut être considérée comme l'une des premières curistes russes dans ce lieu; elle laisse des souvenirs, lettres et autres témoignages (d'après M. BankovskiZüllig, "Zwischen Selbsterfahrung und Fremdeerlebnis. Schweizreisen russischer Schriftstellerinnen um $1860 »$ p. 225-252.

22. Lettre du 9 novembre 1857, citée par C. Ayer, «Notice biographique», p. XV.

23. Ibid., p. XVI.

24. De Kazan, le 9 ou 10 octobre 1858, à sa maîtresse normande, Emma MannouryLacour. Cité par C. Schopp, «Préface», p. 5. 
7 à 8000 fr. par an. Dans l'un et l'autre cas, la besogne est considérablement abrégée et l'on en sort avant d'avoir perdu tous ses cheveux. Tu comprends bien que, si jamais j'arrive au bienheureux chiffre de mes modestes désirs, je n’aurai rien de plus pressé que de revenir au perchoir pour y chanter du soir au matin ${ }^{25}$.

Le $1^{\text {er }}$ juin 1858, il prend un nouveau préceptorat dans une famille dont on ignore le nom, à une lieue d'Odessa.

C'est de mon nouveau logement, au milieu du frémissement des arbres et des préludes du rossignol auxquels le bruit continu de la mer Noire sert d'accompagnement, que je t'écris. Nous sommes dans la plus belle campagne des environs d'Odessa; je n'ai que deux élèves, et on me recommande sans cesse de ne pas les faire trop travailler ${ }^{26}$.

Après une année, las de cet exercice, "son humeur aventureuse le reprend» et "pour tenter la fortune, il va s'établir comme maître d'hôtel à Yalta, sur la côte sud de la Crimée » ${ }^{27}$, en compagnie d'un professionnel zurichois de la branche. Cinq ans après la guerre de Crimée, la province ayant retrouvé le calme, Sciobéret exulte à nouveau:

Tu me demanderas comment je me suis fourré dans cette galère. C'est bien simple, mon cher. J'étais embêté d'être toujours dans la maison d'un autre, d'être le salarié, le valet, la victime de toute espèce de caprices. J'ai voulu essayer de la domination à mon tour, jouir du plaisir d'être le maître, et me voilà dans un pays superbe, avec le climat de Nice, des montagnes derrière et la mer devant, un pays de touristes et de baigneurs; un hôtel de saison où l'on écorche beaucoup, mais pas longtemps. Ferons-nous des affaires (nous sommes deux, un vrai maître d'hôtel de Zurich et moi) ? je l'espère, mais je ne te le dirai qu'après l'été. En attendant je travaille comme un nègre au jardin, à la cave, partout, et je me porte bien et je suis content ${ }^{28}$.

Une saison le déçoit. Bourse plate, il se sépare de son associé dont le caractère trop absolu ne lui plaisait pas. A fin 1860, Sciobéret gagne Tiflis:

25. Cité par C. Ayer, «Notice biographique», p. XV-XVI.

26. Ibid., p. XVII.

27. Ibid., p. XIX.

28. Lettre du 28 mai 1860, ibid., p. XIX. 
J'ai jeté par-dessus les toits la comptabilité du maître d'hôtel, et me voilà à Tiflis gros Jean comme devant, c'est-à-dire enseignant le français à toute espèce d'Asiatiques. Encore si j’avais trouvé le Pérou! mais les pierres sont dures en Asie comme partout ailleurs. Rien ne serait plus facile que de s'établir ici d'une manière avantageuse, mais tout cela ce sont des profits à longue échéance, et je n'ai pas envie de prolonger au-delà d'une certaine mesure mon séjour en Russie. Il faut bien faire une fin, je voudrais vivre avec mes vieux parents, égayer leur vieillesse et leur procurer un peu de bien-être après les rudes travaux qu'ils ont eu à subir. Mais pour cela, tu le comprends du reste, il me faut une certaine somme dont je puisse vivre, je ne dis pas sans travailler, mais moyennant un travail conforme à mes goûts et à mes facultés. Bref, tu vois où j'en suis, à la poursuite d'un but qui n'est pas une chimère, mais qui le sera peut-être pour moi, grâce à mon caractère turbulent et versatile ${ }^{29}$.

Puis, Sciobéret décline deux offres d'enseignement régulier et préfère s'engager comme précepteur à Tsinondale, au nord-est de Tiflis, dans la province de Kakhétie. C'est la partie la plus intéressante de son séjour et aussi la moins documentée:

J'avais trois places à ma disposition, une à Porrentruy, que m'offrait M. Stockmar; une autre au gymnase de Tiflis; une troisième en province, à Tsinondali, chez la princesse Tchavtchavadzé, la captive de Shamyl. Pour fuir les séductions et les dépenses de la ville, j’ai choisi cette dernière, et je pars dans quelques jours pour aller commencer et finir, si possible, l'éducation du petit prince ${ }^{30}$.

Au début de l'année 1861, Sciobéret entre en effet au service du prince David et de la princesse Anna Tchavtchavadzé pour éduquer leur fils Alexandre. Anna et sa sœur, la princesse Varvara Orbeliani, étaient petites-filles de Georges XII, dernier roi de Géorgie (1798-1800), et dames d'honneur de l'impératrice de Russie. Le "château» de Tsinondale, situé au bord d'une rivière affluente du fleuve Alazan dans une région de vignobles et de vergers, avait été conçu par le prince Alexandre Tchavtchavadzé (1786-1846) comme une résidence d'été pour échapper à la poussière de Tiflis. Le prince Alexandre, officier de renom, fut également un poète célèbre. La haute société russe et

29. Lettre du 3 janvier 1861, ibid., p. XX.

30. Lettre du 20 avril 1861, ibid., p. XX-XXI. 
géorgienne de Tiflis venait lui rendre visite à Tsinondale, Lermontov y avait même écrit son poème Le Démon ${ }^{31}$. Quant à son fils, David Tchavtchavadzé, il était l'un des commandants de l'armée russe.

Pierre Sciobéret a donc séjourné dans l'une des familles les plus puissantes de Géorgie. Une famille, en outre, qui fut durement éprouvée avant l'entrée de Sciobéret à son service. Au début de juillet 1854, Anna Tchavtchavadzé fut enlevée en même temps que toutes les femmes et les enfants résidant à Tsinondale par un groupe de musulmans lezghiens à la tête duquel était Ghazi Mohamed, le second fils de l'imam Chamil (1797-1871) ${ }^{32}$. L'enjeu de ce raid était l'échange des otages contre le fils aîné de Chamil, Djamal-Eddine, qui était tombé aux mains des Russes. Anne Drancey, la nounou française qui s'occupait des deux filles de la princesse Tchavtchavadzé, également enlevée puis libérée contre rançon avec tout le groupe, raconte ses souvenirs dans un livre paru en $1857^{33}$. Cet épisode avait fait grand bruit en Europe ${ }^{34}$. Alexandre Dumas, qui est allé en recueillir le récit de la bouche d'Anna Tchavtchavadzé, durant l'hiver 1858-1859, le relate dans le journal de son voyage au Caucase ${ }^{35}$.

3I. Lermontov était lieutenant dans le régiment des dragons de Nijni-Novgorod, stationné en Géorgie, commandé par le même Alexandre Tchavtchavadzé.

32. Chamil, le héros de la résistance des Montagnards contre l'occupant russe. Merci à Jean-Pierre Vauthey de nous rappeler que l'«imam» (celui qui est devant), comparable à l'herméneute, est l'interprète de la parole (du prophète) en lieu et temps, plutôt que le propagateur d'une doctrine.

33. Souvenirs d'une Française captive de Chamyl, recueillis par Edouard Merlieux, Paris, Sartorius, 1857. Réédité sous la forme suivante: A. Drancey, Captive des Tchétchènes. Une traduction russe de l'édition de 1857 par Kiril Dziubinskii est parue en 1858 à Tiflis (Plennitsi Shamilia...).

34. Le rédacteur en chef de la Gazette de Tiflis avait aussi reconstitué les faits à partir de nombreux témoignages: Evgraf Alexeievitch Verderevski, Plennye ou Chamilia pravdivaïa povest o vosmimessiatchnom i chestidnevnom prebyvanii ou Chamilia. Cet article fut recyclé à Paris par H. Delaveau, "Histoire de la captivité de deux princesses russes dans le sérail de Chamyl au Caucase en 1855, d'après le récit russe de M. Verderevski», p. 15. Il existe aussi une traduction en anglais de H. Sutherland Edwards, Captivity of Two Princesses in the Caucasus, [London, 1857].

35. Dumas fait imprimer sa relation à peine plus d'un mois après son retour à Paris, le 10 mars 1859, sous forme de livraisons dans un quotidien expressément édité: Le Caucase. Journal de voyages et romans paraissant tous les jours, Blot (imprimeur), Delavier (éditeur), 16 avril -15 mai 1859. L'édition de librairie suit aussitôt: Le Caucase, Librairie de la France théâtrale, s. d. [1859], 2 vol. (voir Bibliographie de la France, 21 mai 1859). Réédité sous le titre Voyage au Caucase, ill. Jean-Pierre Moynet et Prince Gagarine, préf. Claude Schopp, Hermann, 2002. 
En 1861, Pierre Sciobéret n'arrive donc pas au meilleur moment de l'histoire de la famille Tchavtchavadzé. Le "petit prince» dont il a la charge d'éducation avait été aussi enlevé de Tsinondale, en 1854, alors qu'il était âgé d'un peu plus d'un an.

Il donnait des leçons au prince Tchavtchavadzé, Alexandre, un enfant de près de dix ans, très intelligent, au regard étrangement fixe qui avait l'air de toujours fouiller un horizon lointain. La manière dont, plus tard, l'élève parla le français, témoignera en faveur de la bonne méthode du maître ${ }^{36}$.

Où Robert Loup a-t-il puisé ces informations (aurait-il eu accès à de la correspondance?), car l'éditrice moderne des souvenirs d'Anne Drancey s'étend moins:

Quant au petit prince Alexandre, personne ne sait ce qu'il est devenu, il n'y a plus trace de lui après son retour en Géorgie, quoiqu'il ait été nommé page par l'empereur Alexandre ${ }^{37}$.

Cependant, de son séjour princier, Sciobéret nous apprend seulement que des "désagréments" l'empêchent de persévérer dans son travail (l'écriture) :

Je suis honteux d'avoir été si longtemps sans répondre à ta dernière lettre. Le motif principal a été que je t’avais pour ainsi dire promis, puisque tu avais eu l'obligeance de me trouver un éditeur, de terminer mon dernier-né ${ }^{38}$, et je comptais pour cela sur l'hiver que j’allais passer à Tiflis. Mais, contrairement à mon attente, au lieu du repos et du calme que j'espérais, j'y ai éprouvé tant d'ennuis et tant de désagréments que je n'ai pu venir à bout d'écrire une seule ligne. Aussi ai-je hâte de boucler mes comptes et de reprendre le chemin de l'Occident ${ }^{39}$.

36. R. Loup, Pierre Sciobéret, 1830-1876. Sa vie. Son ceuvre, p. 87.

37. "Postface» de C. F. Herrmann dans A. Drancey, Captive des Tchétchènes, p. 187.

38. Il ne s'agit pas d'Abdallah Schlatter mais de Denney et Tapolet, un roman à caractère social dans le décor fribourgeois. Après en avoir écrit les deux premières parties en Russie, publiées dans la Revue Suisse et Le Confédéré en 1859-1860, il n'en produira jamais la fin.

39. Lettre du 23 août 1863, citée par C. Ayer, «Notice biographique», p. XXII. 
La dernière lettre de Sciobéret est datée du $1^{\text {er }}$ février 1864 à Tiflis. Elle fait le bilan de sept années d'exil et montre que l'homme s'illusionne encore sur son sort financier:

Il est une idée, mon cher, à laquelle j’ai tout sacrifié, celle de l'indépendance. C'est elle qui m’a chassé de mon pays, c'est elle qui m’a empêché d'y rentrer. Tu ne te figureras jamais toutes les privations que j'ai subies, tous les déboires que j’ai supportés pour cette dure maîtresse. Dans quelques mois, s'il plait à Dieu, je l'aurai soumise: j’aurai mon pain sur la planche, et je serai tranquille sur mon avenir ${ }^{40}$.

C'est tout ce qu'on peut savoir - pour l'instant? - des années «caucasiennes" de Sciobéret. Au mois de juillet 1864, après avoir appris le décès de son père, Sciobéret rentre en Suisse, accompagné d'une gouvernante de la famille Tchavtchavadzé, Emélie Ittel, trente-deux ans, fille d'un officier de cuirassiers de Strasbourg, qu'il épousera à Schiltigheim (Bas-Rhin), le 23 septembre 1864.

Quant à Abdallah Schlatter, d'après les témoignages de l'entourage de Sciobéret, rapportés de seconde main, l'histoire aurait été composée après le retour de l'auteur en Suisse. Une lettre datée de 1861 révèle pourtant le vif intérêt que Sciobéret portait déjà aux contrées et aux peuples qu'il découvrait, ainsi que son intention d'en faire un livre:

J'aurais mille détails à t'écrire sur le Caucase, sur les beautés géorgiennes et arméniennes ${ }^{41}$, sur les mœurs, les coutumes, la manière de vivre des indigènes; mais cela demanderait tout un volume, je le réserve pour la Revue suisse, puisque tant est qu'elle tient à moi, seulement il faut que j'aie du temps et surtout l'envie d'écrire ${ }^{42}$.

Ce passage fait supposer des voyages entrepris en-dehors des lieux dont il est question dans les lettres et un contact approfondi avec les populations indigènes. Rien n'est moins sûr: Tiflis, alors capitale culturelle et cosmopolite de la région caucasienne, rassemblait tous les traits mentionnés ci-dessus. C'est finalement au récit d'Abdallah Schlatter que l'on pourra mesurer la connaissance non négligeable que Sciobéret avait du

40. Lettre du $1^{\text {er }}$ février 1864 , ibid., p. XXII.

4I. Il n'est pas sûr qu'il s'agisse de la beauté féminine, la population et la culture arméniennes ayant été fortement implantées en Géorgie à cette époque.

42. Lettre du 20 avril 1861, citée par C. Ayer, «Notice biographique», p. XXI. 
Caucase, avec pour preuve l'étendue géographique qu'il représente, la matérialité socioculturelle qu'il suggère, et les discours politiques qu'il recouvre.

Dans Abdallah Schlatter, Pierre Sciobéret raconte en effet l'aventure du héros éponyme, un jeune homme de vingt-cinq ans, "Suisse d'origine et Turc de nom", représentant de commerce d'une maison de Trébizonde ${ }^{43}$, qui tente de négocier avec les populations de Mingrélie ${ }^{44}$ et d'Abkhazie ${ }^{45}$. A Redout-Kalé ${ }^{46}$, Schlatter rencontre l'Arménien Emphidiantz, un rusé personnage qui tente de s'associer le "Frenghi» dans une opération d'exploitation des richesses naturelles de l'Abkhazie. A cet effet, il suffirait de nouer connaissance avec un prince local et de gagner sa confiance. Mais l'histoire tourne court lors d'un banquet que donne ce chef abkhaze en l'honneur des étrangers: dans son ivresse, Schlatter accepte d'épouser l'une des filles du chef. Dégrisé le lendemain, il est obligé d'assumer son acte inconscient. Le prince abkhaze avait son idée derrière la tête: Schlatter paraîtrait à son peuple l'homme des

43. Trébizonde (en français) = Trapezunt (en allemand), aujourd'hui Trabzon, ville portuaire située sur la rive sud-est de la mer Noire en Turquie dans la province homonyme.

44. Région du nord-ouest de la Géorgie, la Mingrélie était alors un Etat indépendant, néanmoins soumis à la Russie depuis 1801 .

45. Le territoire d'Abkhazie est composé de vallées et de montagnes à l'extrême ouest de la Géorgie, sauf le revers septentrional du Caucase au nord-est qui étend ses plages au bord de la mer Noire. Anciennement, cette région faisait partie du royaume de Colchide (IX $-\mathrm{VI}^{\mathrm{e}}$ siècles av. J.-C.), puis fut colonisée par les Grecs, et par l'empire romain au $\mathrm{I}^{\mathrm{er}}$ siècle apr. J.-C. Les Abkhazes sont convertis au christianisme sous Justinien $I^{\text {er }}$ et deviennent sujets autonomes de l'Empire byzantin jusqu'au IX ${ }^{\mathrm{e}}$ siècle, rattachés ensuite au royaume géorgien d'Iméréthie puis au royaume de Géorgie. Avec la conquête de l'Empire ottoman au XVI' siècle, les Abkhazes sont partiellement convertis à l'islam. Les Géorgiens chassent ensuite les Ottomans et l'Abkhazie devient une principauté autonome sous la dynastie des Sharvashidzé. Au XIXe siècle, les Russes conquièrent graduellement l'Abkhazie entre 1829 et 1842, l'abolition de la principauté n'intervient qu'en 1864. Les Abkhazes de religion musulmane émigrent alors en masse vers l'Empire ottoman... L'Abkhazie est faite "République autonome» au sein de la Géorgie durant l'époque soviétique. Elle proclame son indépendance du pouvoir géorgien en août 1992, indépendance reconnue depuis lors par la Russie suite aux événements de juillet-août 2008.

46. "Redout-Kalé, petite ville fortifiée, possède le port le plus fréquenté de la côte [de la Mingrélie]. Il y entre annuellement 130 à 150 navires. Elle n’a que 500 habitants; mais elle peut devenir importante sous le gouvernement russe.». C. Malte-Brun, Précis de Géographie universelle ou description de toutes les parties du monde [etc.], p. 78. 
entreprises nouvelles face aux envahisseurs russes, alors que le prince lui-même pressent déjà l'inutilité de la résistance et préfère composer avec l'ennemi pour sauver sa vie. Schlatter lui apprendra les manières européennes et, quand la Russie expropriera ses états, le chef abkhaze ira vivre à l'étranger avec une pension à titre de dommages et intérêts...

Les premiers temps du mariage se passent en cavalcades, danses, parties de chasse et fêtes multiples. Plus tard, Schlatter prend au sérieux sa tâche de "ministre» mais s'aperçoit bien vite que ses propositions de réformes ne rencontrent aucun écho. De plus, sa femme, à qui il espérait donner des manières civilisées et enseigner l'usage de la langue française, ne satisfait en rien ses exigences. L'ennui s'installe dans son foyer. Pour couronner le tout, les montagnards lui ravissent son fils pour en faire un vrai musulman et le soustraire à son influence. A bout de patience, Schlatter tente de s'évader: il est poursuivi, repris et ramené de force. Par la suite, le chef abkhaze lui garantit la liberté, mais Schlatter n'a pas la force de s'éloigner définitivement, et on le voit errer entre son foyer et les établissements russes de la côte, noyant son chagrin dans la boisson, peut-être dans l'attente de revoir son enfant...

\section{Saint-Gall- NZZ}

Il reste à dire quelques mots sur le nom d'Abdallah Schlatter mentionné en toutes lettres dès la page 3 du récit de Sciobéret, et raccourci dans les premières lignes du livre aux initiales « $\mathrm{M}$ [onsieur] $\mathrm{S}$. »

Quelques-uns d'entre vous, amis lecteurs, se souviendront peut-être encore d'un article qui parut, il n'y a guère qu'une dizaine d'années, dans un journal de Zurich et qui était conçu à peu près en ces termes:

«Une fortune comme l'on n'en rencontre que dans les contes de fées vient d'échoir à un de nos compatriotes. Nous apprenons par une correspondance particulière qu'on a eu la bonté de nous communiquer que M. S., originaire d'un de nos cantons septentrionaux et simple voyageur de commerce, vient d'épouser sur les plages lointaines de l'Asie une princesse jeune et charmante qui lui apporte en dot un pays plus étendu que la Suisse. Nous félicitons sincèrement notre jeune 
compatriote de son bonheur et nous en tirons un heureux augure pour l'avenir de la contrée qu'il sera sans doute appelé à gouverner. ${ }^{47}$

En Suisse romande, personne n'a eu l'occasion ou n'a fait l'effort d'interroger cette introduction pour le moins surprenante. Pour en savoir plus, il fallait chercher du côté suisse alémanique. Il y aura une vingtaine d'années qu'Ursel Kälin a révélé l'existence de cent onze lettres écrites par un certain Abdullah à ses parents ${ }^{48}$. Celles-ci se trouvent dans le fonds Schlatter des archives de la ville de Saint-Gall, depuis qu'un descendant, Theodor Schlatter, les y a déposées, le 3 février $1987^{49}$. Ce précieux témoignage complète et corrige un article dans lequel Lis Gamper, en 1941, déplorait qu'aucun document original n'existât pour confirmer les aventures d'Abdullah Schlatter ${ }^{50}$. Gamper révélait déjà dans ces lignes consacrées aux voyages en Russie du père d'Abdullah, Daniel Schlatter, le lien existant entre un article de presse paru à la fin des années 1850 dans le Neue Zürcher Zeitung (NZZ) et le roman de Pierre Sciobéret ${ }^{51}$. Il s'appuyait sur une brève notice du livre de famille des Schlatter (Schlatterbuch): «Abdullah Schlatter a fait l'objet d'un roman, il a convolé avec une princesse du Caucase ${ }^{52}$. " Ce fait est confirmé par l'une des lettres qu'Abdullah a envoyées à ses parents, dont voici un extrait:

Mes très chers parents!

Après quatre mois d'absence, Dieu soit loué! j’ai pu enfin rentrer sain et sauf et prendre connaissance de votre lettre: j'en déduis que vous êtes également en bonne santé! [...]

47. P. Sciobéret, Abdallah Schlatter ou les curieuses aventures d'un Suisse au Caucase, p. 1.

48. U. Kälin, «Die St. Galler Daniel und Abdullah Schlatter in Südrußland», p. 335363; U. Kälin donne des informations supplémentaires dans «Die Kaufmannsfamilie Schlatter - ein Überblick über vier Generationen », p. 391-408.

49. Kantonsbibliothek St. Gallen Hauptstelle Vadiana, Stadtarchiv, Nachlass Schlatter.

50. L. Gamper, «Der Tatarenschlatter», p. 7. En 1941, le midi de la Russie revenait sur le devant de la scène avec l'avancée des troupes allemandes sur Mariupol.

5I. Le Zürcher Zeitung paraît pour la première fois en 1780; renommé Neue Zürcher Zeitung à partir de 1821. Sciobéret devait maîtriser la langue allemande après son séjour à Berlin.

52. Cité par L. Gamper, «Der Tatarenschlatter», p. 7. Nous traduisons de l'allemand. 
Je suis resté un mois et demi à Tschamschazwa ${ }^{53}$ en Abasie ${ }^{54}$, et j'ai fait une excursion à Junara; lors d'un séjour de quelques jours auprès d'une famille princière, une des premières du pays, je suis tombé amoureux de la plus jeune fille, et - je me suis marié!

Comme je suis resté peu de temps, je n'ai pas pu réunir tous les papiers nécessaires, d'autant plus que le chef du pays, Michal Bey était absent. J'ai donc laissé ma chère femme là-bas [...].

Elle est de religion grecque-orthodoxe; très docile et très douce; je lui ai traduit le Nouveau Testament autant que faire se peut en mingrélien ${ }^{55}$; j'espère surtout la civiliser en peu de temps, car bien qu'elle soit encore très jeune elle est déjà tout à fait mûre. Chez nous, on lui donnerait facilement vingt-deux ans, en réalité elle n'est âgée que de quatorze ans et demi : c'est justement l'âge où elle peut apprendre tout ce qu'il faut pour son développement moral et ses manières. Elle est très adroite aux travaux féminins. Son prénom est Dudu Khan; sa famille, Markani, est la plus puissante et la plus riche après celle du chef du pays: une grande partie de ses énormes possessions et de ses terres m’a été offerte en dot. [etc.] ${ }^{56}$

D'après Lis Gamper, c'est sur la base cette lettre que la $N Z Z$ a bâti son papier: «Die N.Z.Z. hatte an den wie ein Märchen aus tausendundeiner Nacht anmutenden Artikel die Bemerkung angefügt, sie habe ihre Mitteilungen einem Privatbrief aus Trapezunt entnommen ${ }^{57}$.» Et cette lettre précède une autre, datée du 15 janvier 1858, dans laquelle Abdullah se plaint que la presse a fait un scoop de son mariage avec la princesse abkhaze. Une recherche approfondie permettra sans doute de retrouver l'article qui a servi d'intrigue au roman de Pierre Sciobéret.

Quant à Abdullah Schlatter, il était issu d'une famille de commerçants de textiles saint-gallois. Il avait parfait son apprentissage

53. D’après les témoignages de Karatchaïs et Balkars rencontrés près de l’Abkhazie, en novembre 2008, cette localité doit avoir eu quelque importance mais personne ne sait si elle existe encore. Quant à Junara, les mêmes personnes n'en avaient jamais entendu parler.

54. Abkhazie.

55. Certains locuteurs parlaient (parlent encore?) le mingrélien dans des enclaves de l'Abkhazie. Il existe de nombreux dialectes ou sous-dialectes du mingrélien.

56. Extrait de la «Lettre 77» datée du 7 septembre 1857 à Trébizonde, reproduite en langue originale par U. Kälin, «Die St. Galler Daniel und Abdullah Schlatter in Südrußland", p. 354-355. Traduit par moi de l'allemand en français.

57. L. Gamper, «Der Tatarenschlatter». 
de commerce à Constantinople et dans quelques villes turques, pour le compte de deux différentes firmes suisses associées à des négociants locaux, puis avait parcouru de long en large toute la Transcaucasie jusqu'à la mer Caspienne, finissant par mourir en Perse. D'après les coïncidences que l'on trouve entre la fiction et les lettres, Abdullah Schlatter et Pierre Sciobéret se sont rencontrés probablement une fois, brièvement, à Tiflis. L'écrivain en a tiré de nombreux traits biographiques pour la composition du héros éponyme de son récit. Et, en dépit des apparences qui font imaginer que Sciobéret s'est identifié au héros de son histoire, Abdallah Schlatter fait plutôt référence à "Abdullah Schlatter", personnage historique dont Sciobéret n'est pas le double avéré.

\section{Conclusion}

Ces quelques éléments d'analyse à la fois historique et littéraire rendent possible une étude plus précise d'Abdallah Schlatter ou les curieuses aventures d'un Suisse au Caucase.

Il n'est pas innocent d'intituler son livre Abdallah Schlatter à une époque où le Caucase vient d'être conquis par l'Empire russe, au grand dam sans doute des visées colonialistes d'autres puissances (France, Angleterre). Quel regard porte Sciobéret - ou les personnages de son récit - sur le Caucase? Un regard neutre ou personnel, colonial ou anticolonial, plutôt suisse ou plus généralement orientaliste?

Qui est finalement cet Abdallah// Abdullah Schlatter? Personnage moitié fictif, moitié véritable? Il reste étrange que cette simple association d'un prénom oriental avec un nom de famille bien connu à Saint-Gall n'ait provoqué aucune interrogation chez les commentateurs romands et bien peu Outre-Sarine. Si c'est le Röstigraben... L'écart entre l'intention créatrice et la réception par le public est ici énorme. Quand bien même il se trouve un exemplaire d'Abdallah Schlatter ou les curieuses Aventures d'un Suisse au Caucase dans les rayons "Rossika" de la Bibliothèque nationale de Saint-Pétersbourg.

Jean-Christophe EMmenegger

Fribourg et Lyon 


\section{BIBLIOGRAPHIE}

Ayer, C., "Notice biographique", in Scènes de la Vie champêtre, Lausanne, Lucient Vincent, [1882], p. V-XXX.

Bankovski-Züllig, M., "Zwischen Selbsterfahrung und Fremdeerlebnis. Schweizreisen russischer Schriftstellerinnen um $1860 "$, in Fakten und Fabeln: schweizerisch-slavische Reisebegegnung von 18. bis zum 20. Jahrhundert, hrsg. von M. Bankowski [et al.], Basel/Frankfurt am Main, Helbing \& Lichtenhahn, 1991, p. 225-252.

Bondallaz, P., "Le Mouvement littéraire en pays fribourgeois vers 1850 ", Annales fribourgeoises, 7 (1919), p. 13-21.

Bornet, L., "Revue bibliographique: Scènes de la vie gruyérienne par M. P. Sciobéret", in L'Emulation, Fribourg, Schmid, 1854, p. 248-250.

BRÜLHART, F. J., Etude historique sur la Littérature Fribourgeoise depuis le moyen âge à la fin du XIX siècle, Fribourg, Imprimerie Saint-Paul, 1907.

Buchon, M., "Causeries littéraires», in L'Emulation, Fribourg, Schmid, 1854, p. 85-86.

Delaveau, H., "Histoire de la captivité de deux princesses russes dans le sérail de Chamyl au Caucase en 1855, d'après le récit russe de M. Verderevski ", Revue des deux mondes, $1^{\text {er }}$ mai 1856, p. 15.

Devaud, E., "Les écrivains gruyériens de L'Emulation", Revue de la Suisse catholique, 1900, p. 717-754.

Drancey, A., Captive des Tchétchènes, C. F. Herrmann (éd.), Paris, Mercure de France, 2006.

Gamper, L., "Der Tatarenschlatter», Neue Zürcher Zeitung, und schweizerisches Handelsblatt, Zürich, 1653 (19. Oktober 1941), Blatt 7.

Godet, Ph. E., Histoire littéraire de la Suisse française, Neuchâtel/Paris, Delachaux et Niestlé-Fischbacher, 1890, p. 536-537. 
Gremaud, M., "Pierre Sciobéret (1830-1876). Conteur et romancier gruyérien ", L'Echo littéraire: supplément à La Gruyère, 2 (1980).

Gremaud, M. et Cesa, J., Pierre Sciobéret. Colin l'armailli. Le regain. Sur la trace de Pierre Sciobéret (1830 - 1876), Fribourg/Bulle, BCUF - Editions La Sarine - Société des amis du Musée gruérien, 1999.

Humbert, J., La poésie au Pays de Gruyère, Bienne/Paris, Editions du Chandelier, 1947.

—, "Sciobéret, Pierre", in Encyclopédie du Canton de Fribourg, R. Ruffieux (dir.), Fribourg, Office du Livre, 1977, t. 2, p. 501.

KäLIN, U., "Die St. Galler Daniel und Abdullah Schlatter in Südrußland", in Fakten und Fabeln: schweizerisch-slavische Reisebegegnung von 18. bis zum 20. Jahrhundert, hrsg. von M. Bankowski [et al.], Basel/Frankfurt am Main, Helbing \& Lichtenhahn, 1991, p. 335-363.

—, "Die Kaufmannsfamilie Schlatter - ein Überblick über vier Generationen", Schweizerische Zeitschrift für Geschichte/Revue Suisse d'Histoire/Rivista Storica Svizzera, 48 (1998), p. 391-408.

Kohler, P., Guisan, G. et Pidoux, E., Histoire de la littérature française, vol. 3, Lausanne, Payot, 1949.

Loup, R., Pierre Sciobéret, 1830-1876. Sa vie. Son œuvre, thèse présentée à la Faculté des Lettres de l'Université de Genève, Fribourg, Imprimerie Fragnière Frères, 1929.

—, Un Conteur Gruyérien: Pierre Sciobéret, 1830-1876, avec préface de Gonzague de Reynold, Fribourg, Fragnière Frères Editeurs, 1929.

Maeder, A., Gouverneurs et précepteurs neuchâtelois dans l'empire russe (1800-1890), (Cahiers de l'Institut d'Histoire), Neuchâtel, Faculté des lettres de l'Université de Neuchâtel, 1993.

Maggetti, D., "Une littérature populaire?», in Histoire de la littérature en Suisse romande, R. Francillon (dir.), Lausanne, Payot, 1997, t. 2, p. $147-158$.

Malte-Brun, C., Précis de Géographie universelle ou description de toutes les parties du monde [etc.], t. 8, "Description de l'Asie occidentale», Paris, Aimé André, 1835.

Niquille, J., «Robert Loup [etc.] ", Annales fribourgeoises, 18 (1930), p. $90-91$. 
Rambert, E., «Introduction», Sciobéret, P., Nouvelles scènes de la vie champêtre, Lausanne, Lucien Vincent, 1884, p. V-XV.

Reichlen, J., "Pierre Sciobéret», La Gruyère illustrée, 1890, planche VII, p. 11-15.

ReyfF, S. de, "L'“idéal favori” d'Alexandre Daguet ou les pages littéraires de L'Emulation", Cahiers du Musée gruérien, 5 (2005), p. 22-38.

Reynold, G. de, «Les Poètes de la Gruyère», La Montagne, nov.-déc. 1898, p. 158-163; janv. 1899, p. 2-11.

Rime, F., "Pierre Sciobéret», Cahiers du Musée gruérien, 5 (2005), p. 105-111.

Rossel, V., Histoire littéraire de la Suisse romande des origines à nos jours, Genève/Bâle/Lyon/Paris, H. Georg \& Fischbacher, 1891, t. 2.

Rossel, V. et Jenny, H.-E., Histoire de la littérature suisse, Lausanne/ Berne, Payot \& A. Francke, 1910, t. 2.

Rossier, S., "G. de Reynold et L'Emulation, une lecture orientée», Cahiers du Musée gruérien, 5 (2005), p. 131-140.

—, "Robert Loup et Jean Humbert: deux thèses reynoldiennes", Cahiers du Musée gruérien, 5 (2005), p. 141-151.

Schnetzer, A., "Schweizer im Kaukasus: Forscher, Alpinisten und andere Reisende (1801-1939)", in Fakten und Fabeln, schweizerisch-slavische Reisebegegnung von 18. bis zum 20. Jahrhundert, hrsg. von M. Bankowski [et al.], Basel/Frankfurt am Main, Helbing \& Lichtenhahn, 1991, p. 383-432.

Schopp, C., «Préface», Dumas, A., Voyage au Caucase, Paris, Hermann, 2002, p. 1-9.

Sciobéret, P., Abdallah Schlatter ou les curieuses aventures d'un Suisse au Caucase, Lausanne/Neuchâtel, Blanc Imer et Lebet/S. Delachaux, 1870.

Sterroz, J., "Les Poètes de la Gruyère: choix de leurs poésies", La Gruyère illustrée, 6 (1898), p. 89-104.

Vulliemin, L., "Les Suisses en Russie», Revue suisse, 16 (1853), p. 226-242.

WeCK, A.-S. de, Un mouvement migratoire insoupçonné: les Fribourgeoises en Europe centrale et orientale (1860-1914), Mémoire de Licence, Fribourg, [s. n.], 1998. 
Zellweger, R., Les Débuts du roman rustique: Suisse, Allemagne, France, 1836-1856, Paris, E. Droz, 1941. 